\title{
Análise Assintótica do Acoplamento Mútuo entre Aberturas Retangulares ${ }^{1}$
}

\author{
Fernando José da Silva Moreira e José Ricardo Bergmann \\ Centro de Estudos em Telecomunicações \\ Pontifícia Universidade Católica do Rio de Janeiro
}

\begin{abstract}
O problema de acoplamento mútuo em um conjunto de aberturas retangulares em um plano condutor foi formulado em termos da equação integral e da função de Green. Para esta formulação, o cálculo do coeficiente de acoplamento entre modos nas aberturas necessita da solução numérica de integrais duplas acarretando um enorme esforço computacional. Neste trabalho, uma expansão assintótica da funçāo de Green é introduzida nesta formulação permitindo obter expressões analíticas para estes coeficientes de acoplamento. Estas aproximaçōes são avaliadas através da comparação dos valores de acoplamento entre os modos TE10,01 em duas aberturas separadas obtidos através das expressões exatas e aproximadas. Nesta comparação são consideradas diversas posições relativas entre as aberturas.
\end{abstract}

\begin{abstract}
Asymptotic formulas are derived for the coupling between modes in separated rectangular waveguides apertures embedded in a ground plane. The values obtained for the coupling coefficients between the $\mathrm{TE}_{10}-\mathrm{TE}_{10}$ and $\mathrm{TE}_{10}$ - $\mathrm{TE}_{10}$ modes in adjacent apertures given by approximate expressions are compared with those given by exact expressions.
\end{abstract}

Palavras Chave: Antenas para Comunicação via Satélite, Conjunto de Alimentadores, Eletromagnetismo Aplicado, Antenas Refletoras, Síntese e Análise de Antenas Refletoras.

\section{Introdução}

Antenas compostas de um refletor parabólico iluminado por um conjunto de alimentadores têm larga aplicação no segmento embarcado de um sistema de comunicação via satélite. Elas permitem a geração de múltiplos feixes e o modelamento do diagrama de radiação da antena para cada um destes feixes através do controle da excitação dos elementos deste conjunto. As recentes especificaçôes para as antenas embarcadas determinam níveis de isolamento acima de $27 \mathrm{~dB}$ para polarização cruzada e para polarização principal em coberturas adjacentes. Para satisfazer tais exigências, é essencial que, na obtenção da relação entre o diagrama destas antenas e a excitação dos elementos, sejam utilizadas técnicas de análise rigorosas para determinar os campos na abertura dos elementos do conjunto.

1 Este trabalho foi desenvolvido no CETUC, PUC - Rio, Rua Marquês de São Vicente 225, CEP 22453, Rio de Janeiro. Ele foi financiado pela TELEBRÁS sob contrato PUC/TELEBRÁS JDPqD-415-91.

Manuscrito encaminhado em setembro de 1992; aceito em novembro de 1992. 
Estes conjuntos de alimentadores são usualmente compostos de guias de onda (retangulares ou circulares) ou cornetas (cônicas ou piramidais), estando suas aberturas distribuídas sob o plano focal do parabolóide. Quando comparadas as distribuições de campo encontradas para os elementos isolados, a proximidade das aberturas faz com que as distribuições no interior dos elementos sejam afetadas pelos efeitos de acoplamento mútuo, os quais, além de influir no diagrama de radiação do conjunto, alteram a impedância de entrada destes elementos vista dos circuitos excitadores. Assim, para uma predição adequada do desempenho destas antenas é essencial que a análise dos campos gerados por estes conjuntos considere os efeitos de acoplamento.

Para o caso de guia circulares, a Referência [1] apresenta uma análise exata do acoplamento entre aberturas circulares situadas sobre um plano condutor, utilizando a equação integral e a função de Green. A solução numérica desta equação é obtida expandindo os campos nas aberturas em modos de guias de onda e determinando os coeficientes desta expansão via método de Galerkin. Para as aberturas retangulares, Bird [2] apresentou uma extensão desta técnica na qual as expressões para coeficientes de acoplamento entre os modos envolve integrais quádruplas. A aplicação de método de redução de ordem resulta em expressões envolvendo integrais simples para a auto-admitância e duplas para a mútua $[2,3]$.

Em geral, cornetas piramidais são utilizadas como elementos destes conjuntos, sendo construídas com um pequeno ângulo de abertura a fim de evitar a geração de modos superiores na garganta [4] (ver Figura 1). Para estas cornetas os campos no seu interior se aproximam daqueles em um guia, permitindo a utilização da análise anteriormente mencionada para determinar a distribuição de campos nas aberturas [2]. A parte crucial da aplicação desta análise e a determinação do coeficiente de acoplamento entre os diversos modos, pois envolve o cálculo numérico de integrais.O desenvolvimento de tais antenas requer a consideração de diferentes geometrias de alimentadores e a análise destas ao longo da banda de operação. Isto envolve o cálculo da matriz de acoplamento para cada uma destas situações, resultando em um considerável esforço computacional. Como primeiro objetivo deste trabalho serão detalhadas todas as expressões envolvidas no cálculo do acoplamento entre modos em aberturas retangulares. A seguir, a fim de tornar mais eficiente este cálculo, seguir-se-a passos semelhantes aos desenvolvidos em [1], onde aproximações assintóticas para a função de Green no espaço livre são introduzidas na análise para o acoplamento mútuo, permitindo a solução analítica das integrais. Para avaliar estas aproximações, serão consideradas duas expansões com diferentes erros de truncamento. Os resultados obtidos para o acoplamento do modo fundamental são comparados aos fornecidos pelas expressões exatas. 


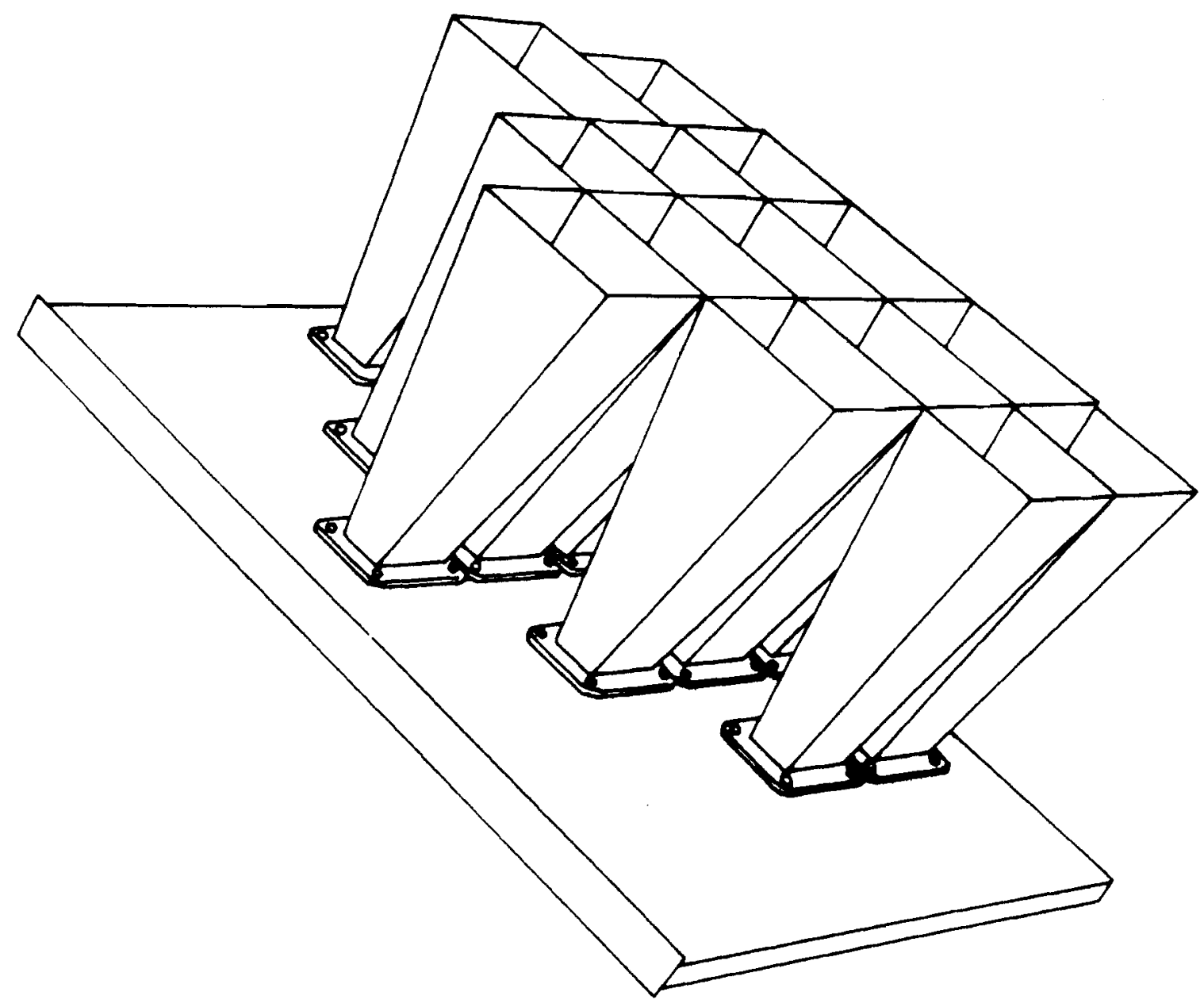

Figura 1 - Conjunto de cornetas piramidais

\section{Acoplamento Mútuo entre os Campos nas Aberturas dos Alimentadores}

A formulação a ser apresentada foi inicialmente desenvolvida por Bird [1] e supõe que as diversas aberturas dos alimentadores (guias ou cornetas) estejam situadas num plano condutor elétrico infinito. Na Figura 2 e apresentada a geometria destas aberturas sobre o plano condutor $(z=0)$, juntamente com os sistemas de coordenadas a serem utilizados. Estas aberturas são retangulares e seus lados são supostos paralelos ou ao eixo cartesiano $\mathrm{x}$ ou ao $\mathrm{y}$. A formulação leva em conta uma variação temporal da forma $\exp (+\mathrm{j} \omega t)$ para os campos.

Devido a presença do plano condutor podemos aplicar o Princípio da Equivalência e o Método das Imagens, resultando na substituição deste plano e das aberturas dos alimentadores por uma nova distribuição de correntes elétrica $\left(\mathrm{J}_{\mathrm{S}}\right)$ e magnética $\left(\mathrm{M}_{\mathrm{s}}\right)$ : 


$$
\mathrm{J}_{S}=0 ; M_{S}=-2 z \times\left. E\right|_{z=0}
$$

onde z é o vetor unitário normal ao plano e E é o vetor campo elétrico total na abertura (incluindo os efeitos de acoplamento mútuo). É importante observar que $\mathrm{M}_{\mathrm{S}}$ é nula fora das regiōes correspondentes as aberturas dos alimentadores em $\mathrm{z}=0$.

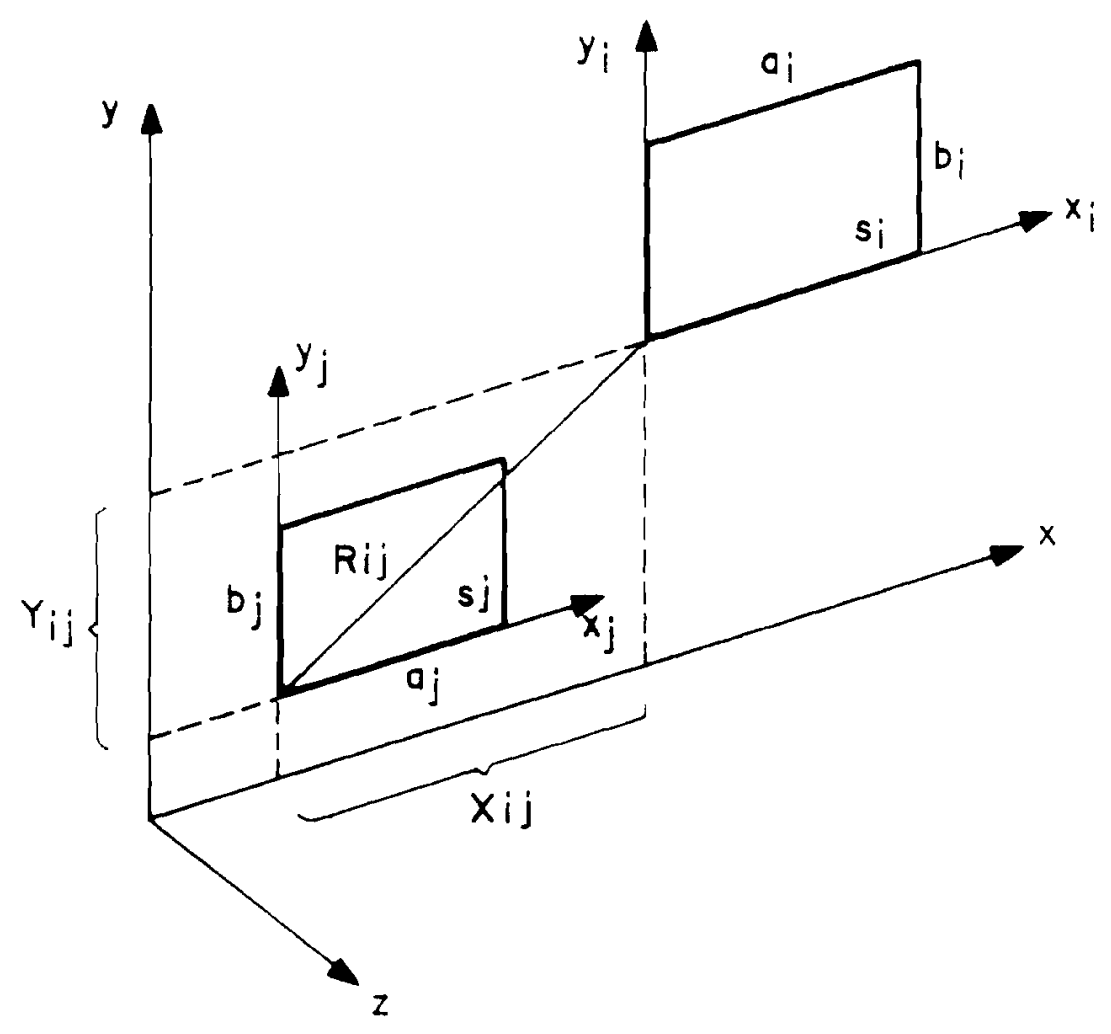

Figura 2 - Geometria das aberturas no plano condutor

A partir da distribuição de correntes sobre o plano, podemos calcular a componente transversa a $z$ do campo magnético radiado a uma distância $R$ da origem [1]:

$$
\begin{gathered}
H_{t}=\frac{j}{2 \pi \omega \mu}\left(K_{0}^{2}+\nabla_{t} \nabla_{t}\right) \cdot z \times \iint_{s} E_{t}\left(s^{\prime}\right) G\left(\left|r-r^{\prime}\right|\right) d s^{\prime} \\
G(\alpha)=\frac{e^{-j K_{0} \alpha}}{\alpha}
\end{gathered}
$$


onde $\mathrm{j}=\sqrt{-1}, \mathrm{~K}_{0}$ é a constante de onda do espaço livre, $\omega=2 \pi$ a freqüência, $\mu$ é a permeabilidade do meio, $r\left(r^{\prime}\right)$ representa a posição do campo (fonte), $\mathrm{E}_{\mathrm{t}}\left(\mathrm{s}^{\prime}\right)$ é a componente transversa a $\mathrm{z}$ do campo elétrico na abertura, e o domínio de integração $\mathrm{S}$ são as regiões das diversas aberturas sobre o plano $z=0$. O operador $\nabla_{t}$ é definido como:

$$
\nabla_{t} \phi=\frac{\partial \phi}{\partial x} x+\frac{\partial \phi}{\partial y} y
$$

onde $\mathrm{x}$,y são os vetores unitários cartesianos. Calculando $\mathrm{H}_{\mathfrak{t}}$ sobre o próprio plano $\mathrm{z}=\mathrm{O}$, podemos aplicar o Método dos Momentos para a solução desta equação aproximando os campos $\mathrm{E}_{\mathrm{t}}$ e $\mathrm{H}_{\mathrm{t}}$ através de uma expansão envolvendo $\mathrm{M}$ modos (TE e TM) de guias retangulares com as mesmas dimensões das respectivas aberturas. Os campos destes modos serão representados por $h_{m}$ (componente tangencial magnética do modo):

$$
\begin{gathered}
H_{t}=\sum_{m}^{M}\left[C_{m} e^{-j v_{m} z}-d_{m} e^{+j v_{m} z}\right] h_{m} \\
E_{t}=\sum_{m}^{M} Z_{m}\left[C_{m} e^{-j v_{m} z}+d_{m} e^{+j v_{m} z}\right] h_{m} \times z
\end{gathered}
$$

onde $\mathrm{v}_{\mathrm{m}}$ é a constante de propagação do modo, $\mathrm{Z}_{\mathrm{m}}$ a sua impedância, e c (d) é a amplitude complexa do modo incidente (refletido). Substituindo as equações (5) e (6) na (2):

$$
\mathrm{H}_{\mathrm{t}}^{\mathrm{i}}=\sum_{\mathrm{m}} \mathrm{B}_{\mathrm{m}}{ }^{\mathrm{i}} \mathrm{h}_{\mathrm{m}}{ }^{\mathrm{i}}=\frac{\mathrm{j}}{2 \pi \omega \mu} \sum_{\mathrm{n}=1}^{\mathrm{M}} \sum_{\mathrm{K}=1} \mathrm{~A}_{\mathrm{n}}{ }^{\mathrm{K}} \mathrm{Z}_{\mathrm{n}}\left(\mathrm{K}_{0}{ }^{2}+\nabla_{\mathrm{t}} \nabla_{\mathrm{t}}\right) \cdot \iint_{\mathrm{S}} \mathrm{h}_{\mathrm{n}}{ }^{\mathrm{K}} \mathrm{G}\left(\left|\mathrm{r}-\mathrm{r}^{\prime}\right|\right) \mathrm{ds}^{\prime} \mathrm{K}^{\prime}
$$

onde o índice " $\mathrm{m}$ " indica o modo na abertura " $\mathrm{i}$ ", o índice " $\mathrm{n}$ " indica o modo na abertura " $k$ ", $A_{n}{ }^{K}=C_{n}{ }^{k}+d_{n}{ }^{K}, B_{m}{ }^{i}=C_{m}{ }^{i}-d_{m}{ }^{i}$ e $S_{K}$ é a área da abertura “ $k$ ". Esta é uma equação integral cujas soluções devem satisfazer as condições de contorno impostas pelas aberturas dos alimentadores, o que é atendido através da utilização dos modos TE e TM. Aplicando o método de Galerkin (multiplicando ambos os lados por $\mathrm{h}_{\mathrm{m}}{ }^{\mathrm{i}}$ e integrando sobre a abertura $\mathrm{s}_{\mathrm{i}}$ ), chega-se a seguinte relação matricial:

$$
\left[\mathrm{B}_{\mathrm{m}}^{\mathrm{i}}\right]=\left[\mathrm{C}_{\mathrm{mn}}{ }^{\mathrm{ik}}\right]\left[\mathrm{A}_{\mathrm{n}}^{\mathrm{k}}\right]
$$




$$
\begin{gathered}
\text { onde } C_{m n}{ }^{i k}=\frac{j}{2 \pi \omega \mu} \frac{Z_{n}}{N_{m}} \iint_{s_{i}} h_{m}{ }^{i} \cdot\left(k_{o}{ }^{2}=\nabla_{t} \nabla_{t}\right) \cdot \iint_{s_{k}} \int h_{n}{ }^{k} G\left(\left|r-r^{\prime}\right|\right) d s_{k}{ }^{\prime} d s_{i} \\
\text { e } \quad N_{m^{*}=} \iint_{s_{i}} h_{m}{ }^{i} \cdot h_{m}{ }^{i} \cdot d s_{i}
\end{gathered}
$$

É mostrado no Apêndice 9.2 da Referência [1] que, aplicando o teorema de Green a equação (9), esta pode ser expressa de uma forma mais conveniente como:

$$
\begin{gathered}
C_{m n}^{i k}=\frac{j w \varepsilon_{0}}{2 \pi} \int_{s_{i}} \int W_{m}^{i} \cdot \int_{S_{k}} \int W_{n}{ }^{K} G\left(\left|r-r^{\prime}\right| d s_{k}\right. \\
W_{m, n}^{i, k}=h_{m, m}^{i, k}+\frac{V_{m, n}}{k_{0}} H z_{m, n}^{i, k} z
\end{gathered}
$$

onde $\varepsilon_{\mathrm{o}}$ é a permissividade do espaço livre e $\mathrm{H}_{\mathrm{Z}}$ e a componente do modo na direção $\mathrm{z}$ (nula para modos TM). No presente estudo as aberturas são retangulares, de forma que serão utilizados os modos TE e TM de um guia retangular com dimensões a,b [5]:

$$
\begin{aligned}
& h=\left\{\frac{j \pi v}{\lambda^{2}}\left[\frac{p}{a} \operatorname{sen}\left(\frac{q \pi x}{a}\right) \cos \left(\frac{q \pi y}{b}\right) x+\frac{q}{b} \cos \left(\frac{p \pi x}{a}\right) \operatorname{sen}\left(\frac{p \pi y}{b}\right) y\right], T E\right.
\end{aligned}
$$

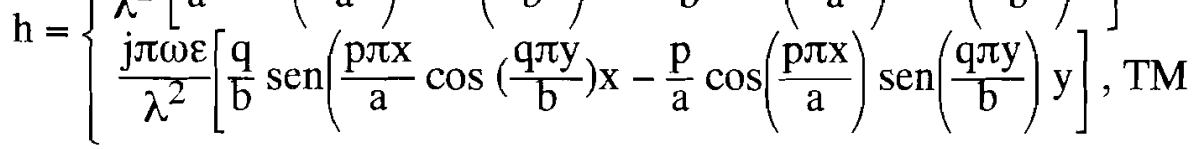

$$
\begin{aligned}
& H z=\cos \left(\frac{p \pi x}{a}\right) \cos \left(\frac{q \pi y}{b}\right), \text { para modos TE } \\
& \lambda^{2}=\left(\frac{p \pi}{a}\right)^{2}+\left(\frac{q \pi}{b}\right)^{2} \\
& \mathrm{v}=\sqrt{\mathrm{k}_{0}^{2} \varepsilon_{\mathrm{r}}-\lambda^{2}}
\end{aligned}
$$

onde $\varepsilon_{\mathrm{r}}=\sqrt{\varepsilon / \varepsilon_{0}}$ é a permissividade relativa do meio. Substituindo as equações (13)(16) nas equaçōes (10)-(12) obtemos as expressóes para o acoplamento entre aberturas retangulares, as quais envolvem integrais quádruplas. 
A relação entre os coeficientes $C_{m}$ dos modos incidentes na abertura com os $d_{m}$ dos refletidos é dada pela matriz [S], a qual pode ser obtida através da seguinte expressão matricial:

$$
\left[\mathrm{d}_{\mathrm{m}}\right]=[\mathrm{S}]\left[\mathrm{C}_{\mathrm{m}}\right] ;[\mathrm{S}]=2\left\{[\mathrm{I}]+\left[\mathrm{C}_{\mathrm{m} \mathrm{n}}^{\mathrm{ik}}\right]\right\}^{-1}-[\mathrm{I}]
$$

onde [I] é a matriz identidade.

0 problema de redução de ordem das integrais quádruplas na expressão do acoplamento entre modos é o ponto crucial tendo sido abordado por vários autores [2-3]. Na referência [2], o emprego do método de Lewin permite reduzir a ordem das integrais na eq.(11) e as expressões resultantes são apresentadas no Apêndice A. Elas envolvem integrais duplas para o acoplamento mútuo e simples para o auto-acoplamento. A determinação da matriz [S] requer a solução numérica de tais integrais, envolvendo um tempo de computação que cresce com o número total de modos utilizados (número de elementos x M)elevado ao quadrado. Mesmo para um número pequeno de clementos este tempo pode ser considerável.

\section{Aberturas Afastadas Entre Si (Expansão Assintótica)}

Para o cálculo do acoplamento entre modos em aberturas circulares, obtém-se significativa redução no tempo de computação dos coeficientes de acoplamento pela utilização fórmulas assintóticas. Estas fórmulas foram obtidas pela introdução, na análise, de aproximação para a função de Green para o espaço livre, as quais permitiram obter expressões analíticas para o acoplamento entre modos em aberturas separadas. Esta redução se torna considerável na medida que é expressivo o número de elementos na matriz [C] derivados do acoplamento mútuo entre modos em diferentes aberturas. Para aberturas retangulares, aproximações assintóticas, similares às empregadas em [2], serão utilizadas para obter fórmulas analíticas para o acoplamento mútuo.

Quando a distância entre as duas aberturas onde os coeficientes de acoplamento estão sendo calculados, for suficientemente grande, a função de Green do espaço livre representada pela equação (3) pode ser expandida da seguinte forma:

$$
\begin{gathered}
G\left(\left|r-r^{\prime}\right|\right) \cong G\left(R_{i k}\right) e^{-j k_{0} f_{1}(\nabla x, \nabla y)} \times\left\{1-\frac{1}{R_{i k}}\left[f_{1}(\nabla x, \nabla y)+\right.\right. \\
\left.+\left(f_{2}(\nabla x, \nabla y)\right)^{2}\left(\frac{j k_{0}}{2}+\frac{1}{2 R_{i k}}-\frac{j k_{0}}{2 R i k} f_{1}(\nabla x, \nabla y)+\frac{k_{0}^{2}}{8 R_{i k}}\left(f_{2}(\nabla x, \nabla y)\right)^{2}\right)\right]+
\end{gathered}
$$




$$
\begin{gathered}
\left.+\frac{1}{\mathrm{R}_{\mathrm{ik}}{ }^{2}}\left(\mathrm{f}_{1}(\nabla \mathrm{x}, \nabla \mathrm{y})\right)^{2}\right\} \\
\left\{\begin{array}{l}
\mathrm{f}_{1}\left(\nabla \mathrm{x}, \nabla \mathrm{y}=\nabla \mathrm{x} \cos \left(\phi_{\mathrm{L}}\right)+\nabla \mathrm{ysen}\left(\phi_{\mathrm{L}}\right)\right. \\
\mathrm{f}_{2}(\nabla \mathrm{x}, \nabla \mathrm{y})=\nabla \mathrm{x} \operatorname{sen}\left(\phi_{\mathrm{\iota}}\right)+\nabla \mathrm{y} \cos \left(\phi_{\iota \mathrm{K}}\right)
\end{array}\right.
\end{gathered}
$$

onde $\nabla \mathrm{x}=\mathrm{x}-\mathrm{x}^{\prime}, \nabla \mathrm{y}=\mathrm{y}-\mathrm{y}^{\prime}, \mathrm{R}_{\mathrm{ik}}{ }^{2}=\mathrm{x}_{\mathrm{ik}}{ }^{2}+\mathrm{y}_{\mathrm{ik}}{ }^{2} \mathrm{e} \phi_{\mathrm{lk}}=\tan ^{-1}\left(\mathrm{y}_{\mathrm{ik}} / \mathrm{x}_{\mathrm{ik}}\right)$ para $\mathrm{x}_{\mathrm{ik}} \mathrm{e}$ $y_{i k}$ representados na Figura 2. Substituindo a equação (18) na (11), todas as integrais podem ser resolvidas analiticamente e a equação (A.2) do Apêndice A passa a ser escrita da seguinte forma (com $C$ dado pela equação (A.1)):

$$
\begin{aligned}
& \underset{\mathrm{z}}{\mathrm{I}} \underset{\mathrm{y}}{\mathrm{x}}=\mathrm{G}\left(\mathrm{R}_{\mathrm{ik}}\right)\left\{\mathrm{E}_{0} \mathrm{f}_{0}-\frac{1}{\mathrm{R}_{\mathrm{ik}}}\left[\mathrm{E}_{1} \mathrm{~F}_{0} \operatorname{cotan}\left(\phi_{\mathrm{L}}\right)+\mathrm{E}_{0} \mathrm{~F}_{1} \tan \left(\phi_{\mathrm{L}}\right)-\right.\right. \\
& \left.-F_{1} F_{1}\left(j k_{0}+\frac{3}{R_{i k}}\right)+\frac{E_{2} F_{0}}{2}\left(j k_{0}+\frac{s_{1}\left(\phi_{\iota k}\right)}{R_{i k}}\right)+\frac{E_{0} F_{2}}{2}\left(j k_{0}+\frac{s_{2}\left(\phi_{\iota k}\right)}{R_{i k}}\right)\right]+ \\
& +\frac{j k_{0}}{R_{i k}^{2}}\left[\operatorname{cotan}\left(\phi_{\iota \kappa}\left(E_{3} F_{0}+E_{1} F_{2} s_{2}\left(\phi_{\iota \kappa}\right)\right)+\tan \left(\phi_{\iota \kappa}\right)\left(E_{0} F_{3}+E_{2} F_{1} s_{1}\left(\phi_{\iota \kappa}\right)\right)\right]\right. \\
& \left.-\frac{\mathrm{k}_{0}^{2}}{8 \mathrm{R}_{\mathrm{ik}}^{2}}\left[\mathrm{E}_{4} \mathrm{f}_{0}+\mathrm{E}_{0} \mathrm{~F}_{4}-4\left(\mathrm{E}_{3} \mathrm{f}_{1}+\mathrm{E}_{1} \mathrm{~F}_{3}\right)+6 \mathrm{E}_{2} \mathrm{~F}_{2}\right]\right\} \\
& \mathrm{s}_{1}(\phi \mathrm{ik})=1-2 \operatorname{cotan}^{2}(\phi \mathrm{ik}) ; \mathrm{s}_{2}(\phi \mathrm{ik})=1-2 \tan ^{2}(\phi \mathrm{ik}) \\
& \mathrm{E}_{\mathrm{L}}=\operatorname{sen}^{\mathrm{L}}(\phi i \mathrm{k}) \times \sum_{\mathrm{i}=0}^{\mathrm{L}}(-1)^{1} \frac{\mathrm{L} !}{1 !(\mathrm{L}-1) !} \underset{\mathrm{c}}{\mathrm{IX}} \mathrm{s}(1, \mathrm{~L}-1) \\
& \mathrm{F}_{\mathrm{L}}=\cos ^{\mathrm{L}}(\phi \mathrm{ik}) \times \sum_{\mathrm{i}=0}^{\mathrm{L}}(-)^{-1} \frac{\mathrm{L} !}{1 !(\mathrm{L}-1) !} \underset{\mathrm{c}}{\mathrm{IY}} \underset{\mathrm{s}}{\mathrm{s}}(1, \mathrm{~L}-1)
\end{aligned}
$$




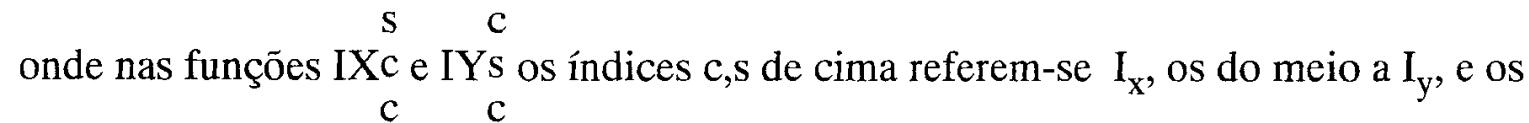
de baixo a $I_{z}$. Estas funções são dadas por:

$$
\begin{aligned}
& \underset{c}{\operatorname{IXc}(t, u)}=\int_{0}^{a_{k}} \int_{0}^{a_{i}} \alpha^{t} \beta^{\mu} \frac{\operatorname{sen}}{\cos l e t}\left(\frac{p_{n} \pi \alpha}{a_{k}} r i g h t\right) \underset{\cos }{\cos }\left(\frac{p_{m} \pi \beta}{a_{i}}\right) e^{j k_{x}(\alpha-\beta)} d \beta d \alpha \\
& I Y_{c}^{s}=\int_{0}^{b_{k}} \int_{0}^{b_{1}} \alpha^{t} \beta^{\mu} \frac{\cos }{\operatorname{sen}}\left(\frac{q_{n} \pi \beta}{b_{1}}\right) \underset{\cos }{\cos } \cos \left(\frac{q_{m} \pi \beta}{b_{1}}\right) e^{i k_{y}(\alpha-\beta)} d \beta d \alpha(25) \\
& \mathrm{K}_{\mathrm{x}}=\mathrm{K}_{0} \cos \left(\phi_{\iota \kappa}\right), \mathrm{K}_{\mathrm{y}}=\mathrm{K}_{0} \operatorname{sen}(\phi \mathrm{ik})
\end{aligned}
$$

onde as integrais em (24) e (25) podem ser resolvidas analiticamente.

\section{Comparação de Resultados Utilizando a Expansão Assintótica entre Aberturas Afastadas}

Para analisar as aproximações apresentadas, será considerado o acoplamento mútuo entre os modos $\left(\mathrm{TE}_{10}\right.$ ou $\left.\mathrm{TE}_{01}\right)$ em duas aberturas quadradas, idênticas e dispostas sobre um plano condutor infinito. As Figuras 3.a-d mostram os valores de acoplamento obtidos pelo uso da formulação integral (-) os quais são comparados com aqueles dados pela expansão assintótica. Para avaliar a convergência da expressão (20), são mostrados valores de acoplamento calculados incluindo até os termos em $1 / \mathrm{R}_{\mathrm{i} \mathrm{k}}{ }^{2} \mathrm{em}$ (18) e que será referido como Exp-1 e representada por (- - -) na figura, e incluindo os termos até $1 / \mathrm{R}_{\mathrm{i} \mathrm{k}}{ }^{3}$ referido como Exp-2 e representado por (.......).

As diferentes posições relativas entre as aberturas consideradas na análise são apresentadas juntamente com as curvas para o acoplamento, onde $\mathrm{W}$ e a dimensão dos lados das aberturas e $\mathrm{D}$ é a distância entre paredes (nestes casos, $\mathrm{D}=0,05 \lambda, 1 \lambda$ e $4 \lambda$ ). Para duas aberturas ao longo do plano $-\mathrm{E}$ ou $-\mathrm{H}$, são considerados na expansão modal (Equação 5) somente aqueles modos (entre os doze primeiros) para os quais existe acoplamento. A utilização de um número maior de modos nesta expansão não altera significativamente os resultados obtidos [6]. Para o caso das aberturas ao lonqo do plano-E, foi utilizada uma expansão modal com 5 modos (TE10, TE11, TM11, TE12, TM12). Para o caso do plano - $\mathrm{H}$, foram utilizados os modos (TE10, TE12, TM12); e para o do plano diagonal, 12 modos (TE10, TE01, TE11, TM11, TE20, TE02, TM21, TM21, TE12, TM12, TE22, TM22). 

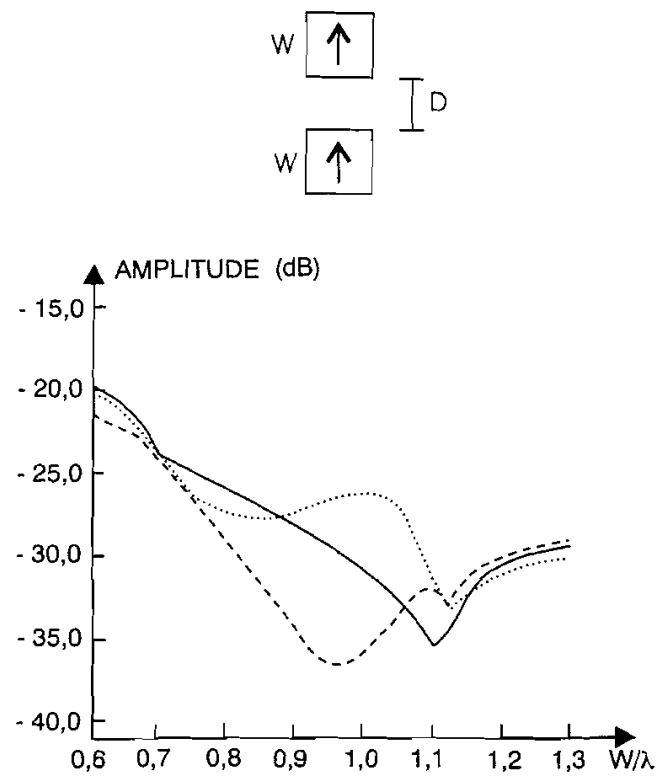

(i)

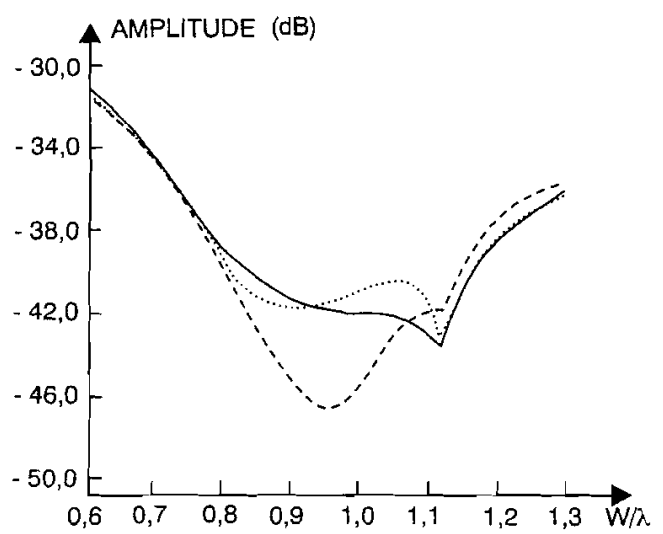

(ii)
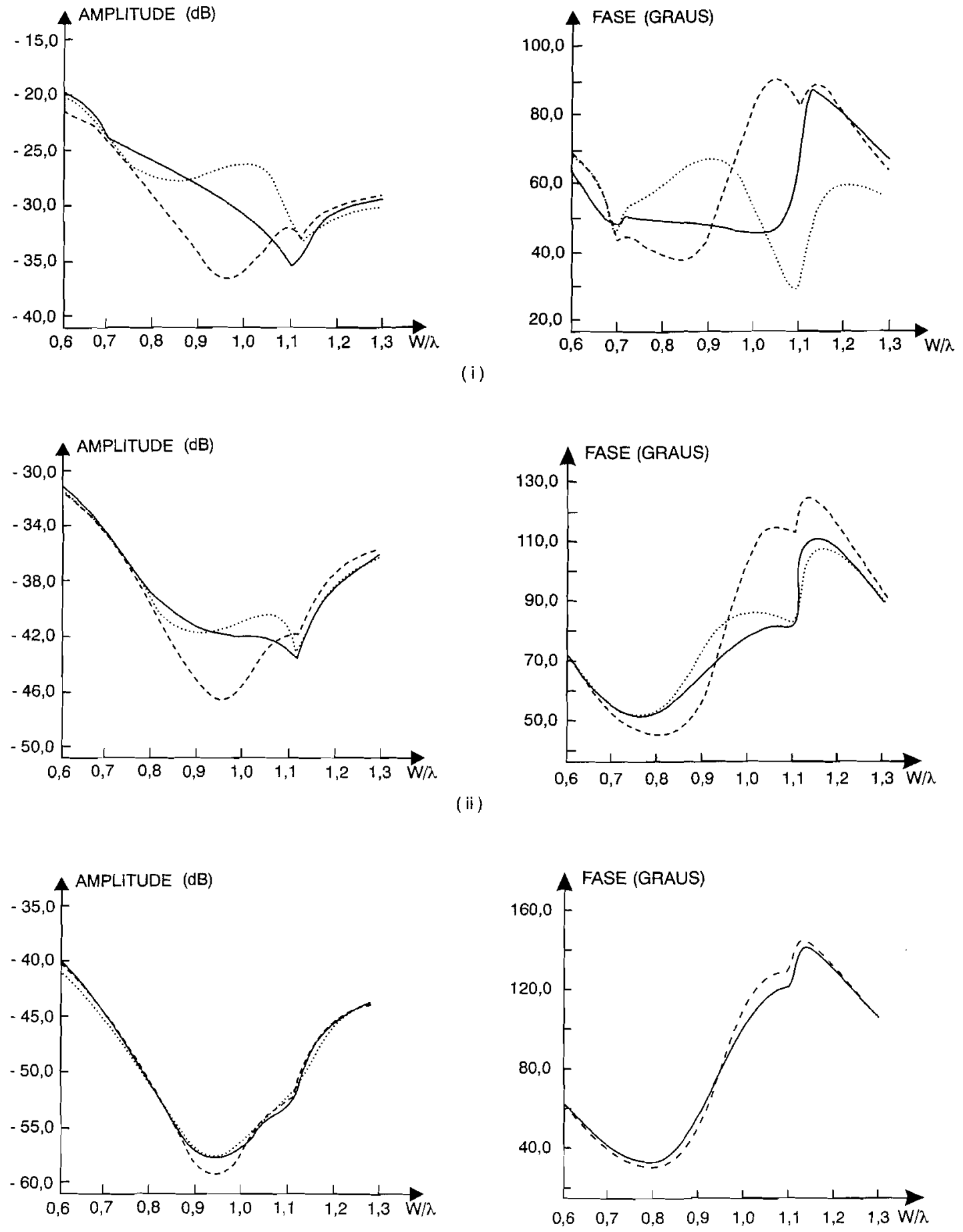

(iii)

Fig. 3a - Acoplamento entre os modos $T E_{10}-T E_{10}$ (plano - E):

(i) $D=0,05 \lambda$; (ii) $D=1 \lambda$; (iii) $D=4 \lambda$ 

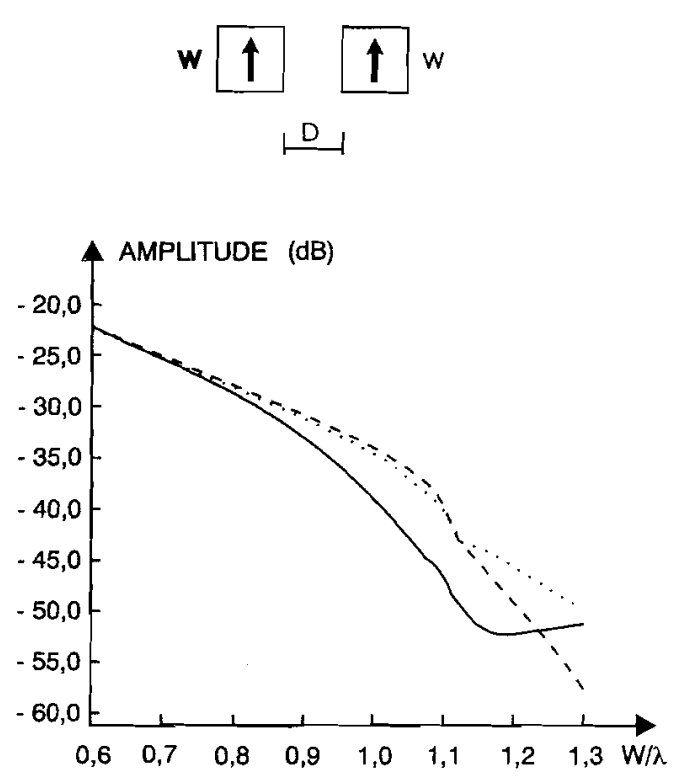

(i)
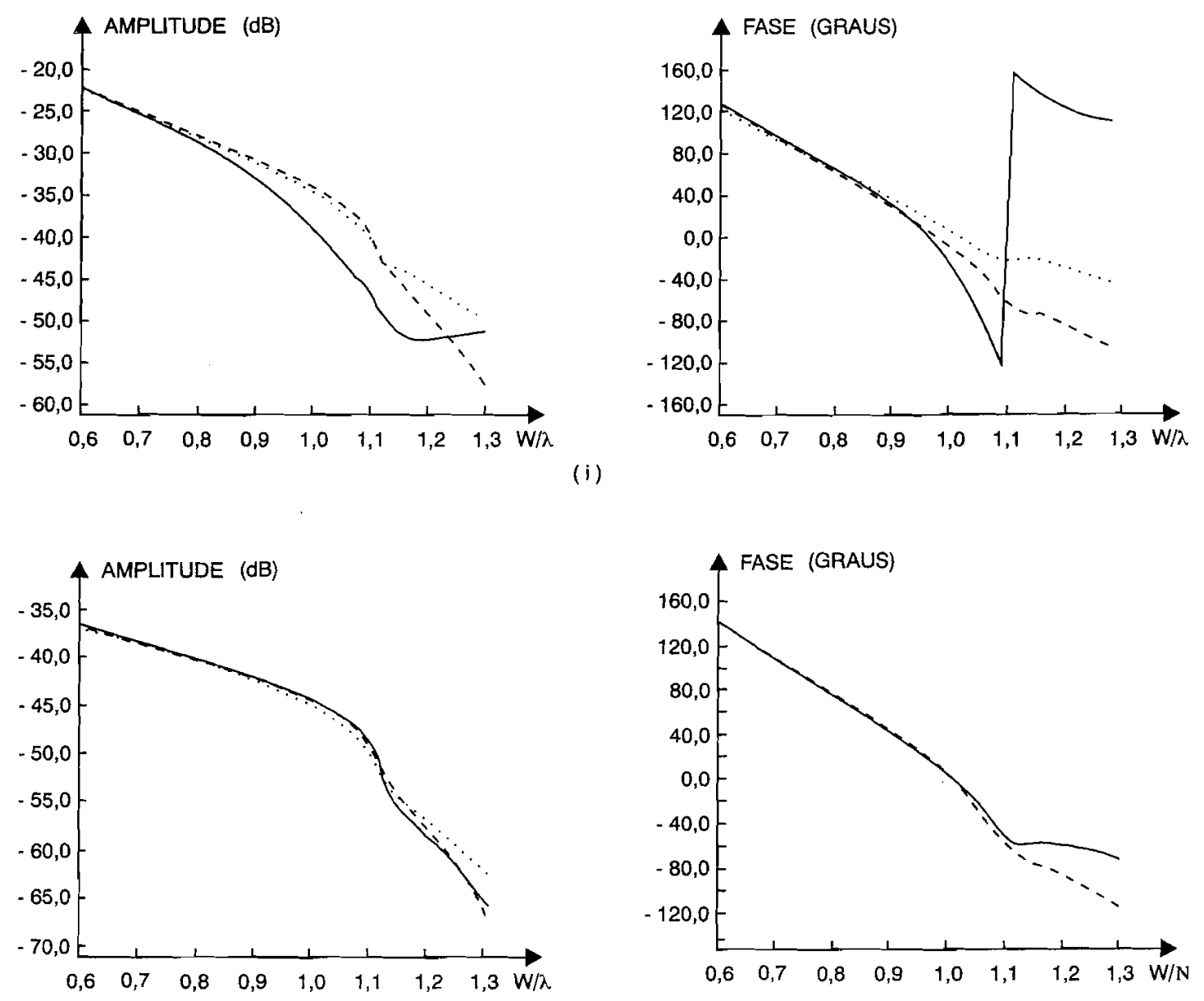

(i)
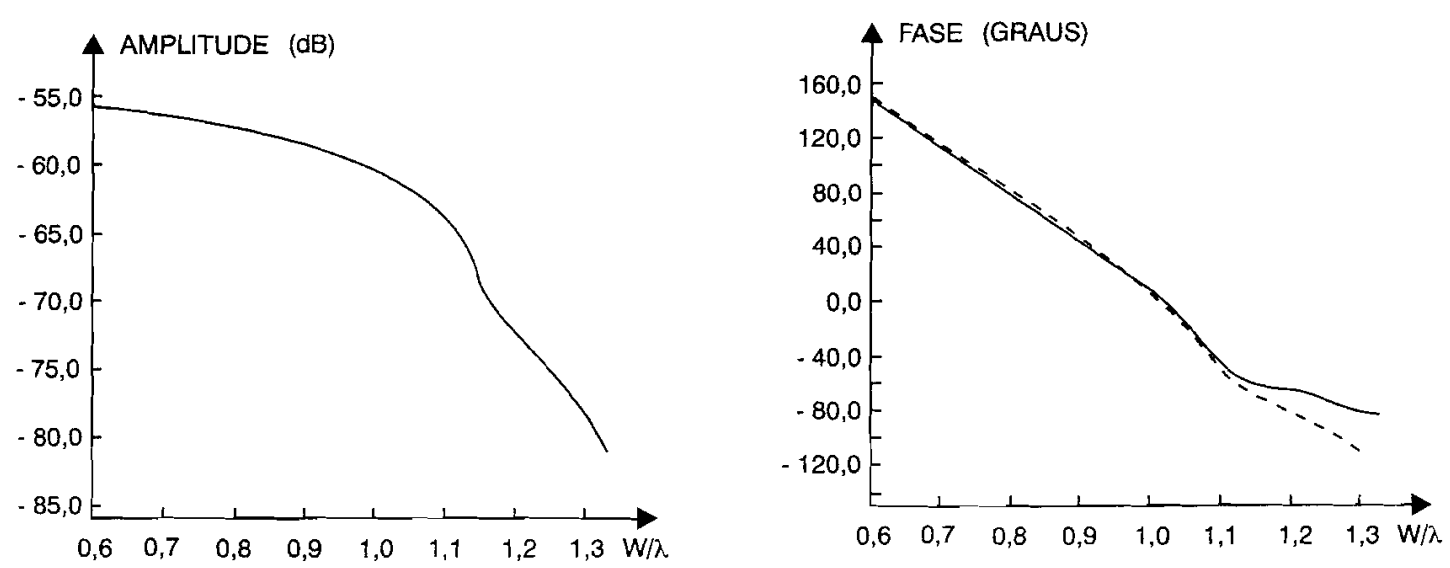

(iii)

Fig. 3b - Acoplamento entre os modos $T E_{10}-T E_{10}$ (plano - H):

(i) $D=0,05 \lambda$; (ii) $D=1 \lambda$; (iii) $D=4 \lambda$ 

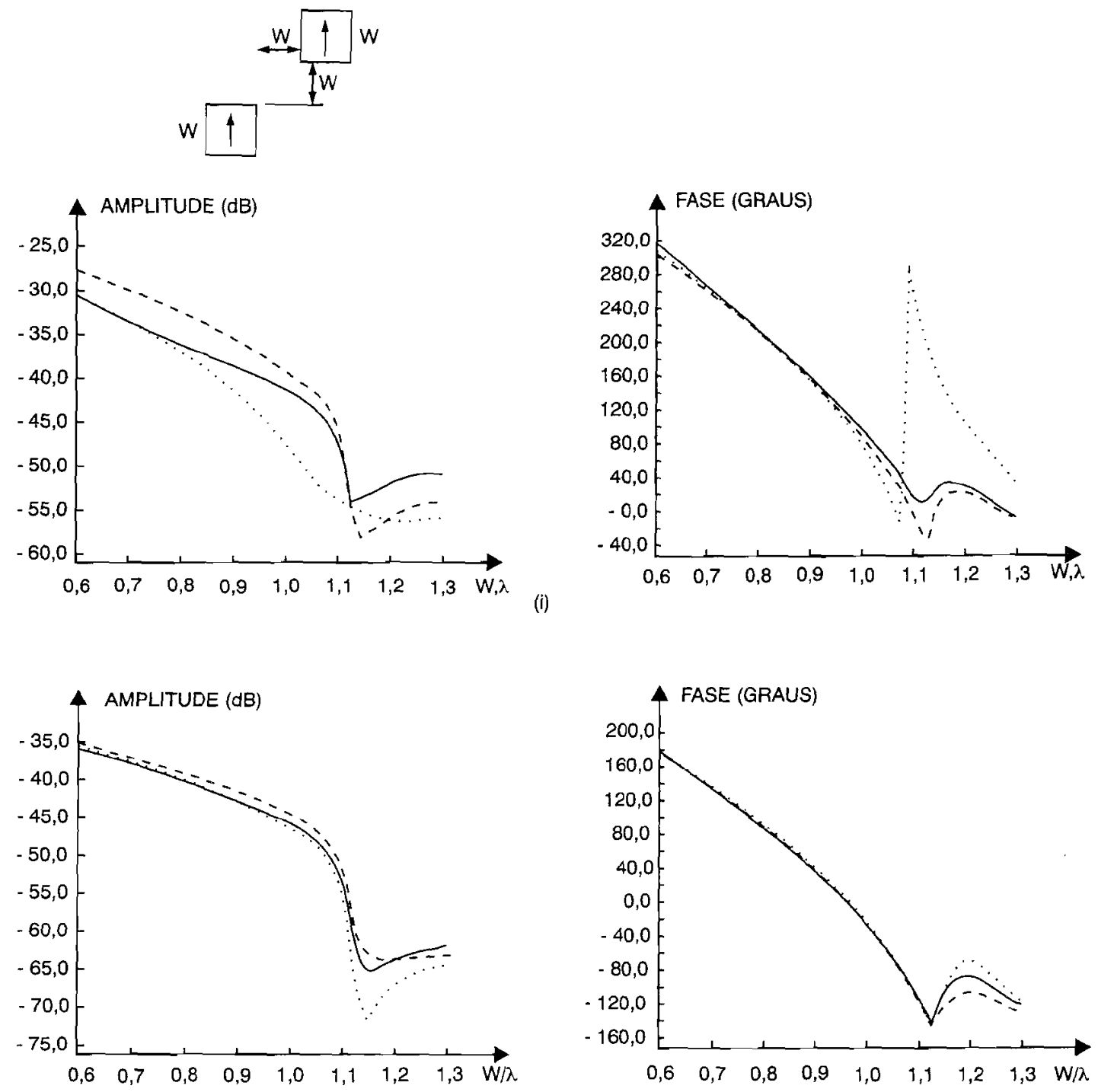

(ii)
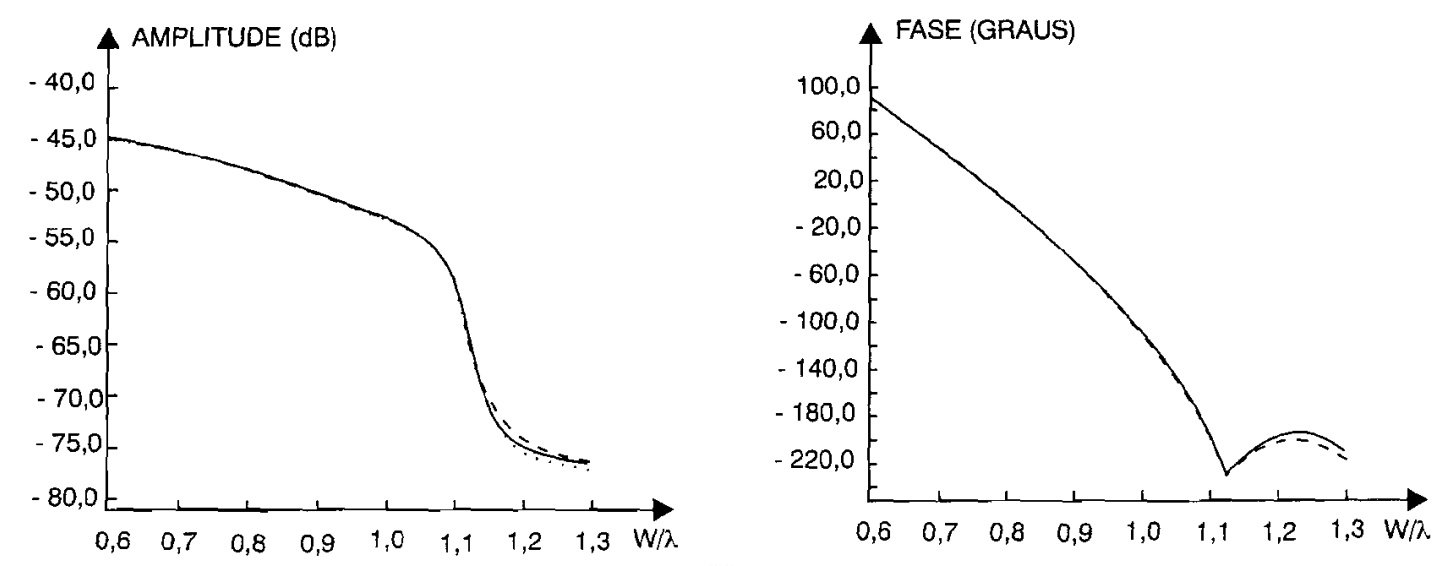

Fig. 3c - Acoplamento entre os modos $T E_{10}-T E_{10}$ (plano diagonal): (i) $D=0,05 \lambda$; (ii) $D=1 \lambda$; (iii) $D=4 \lambda$ 

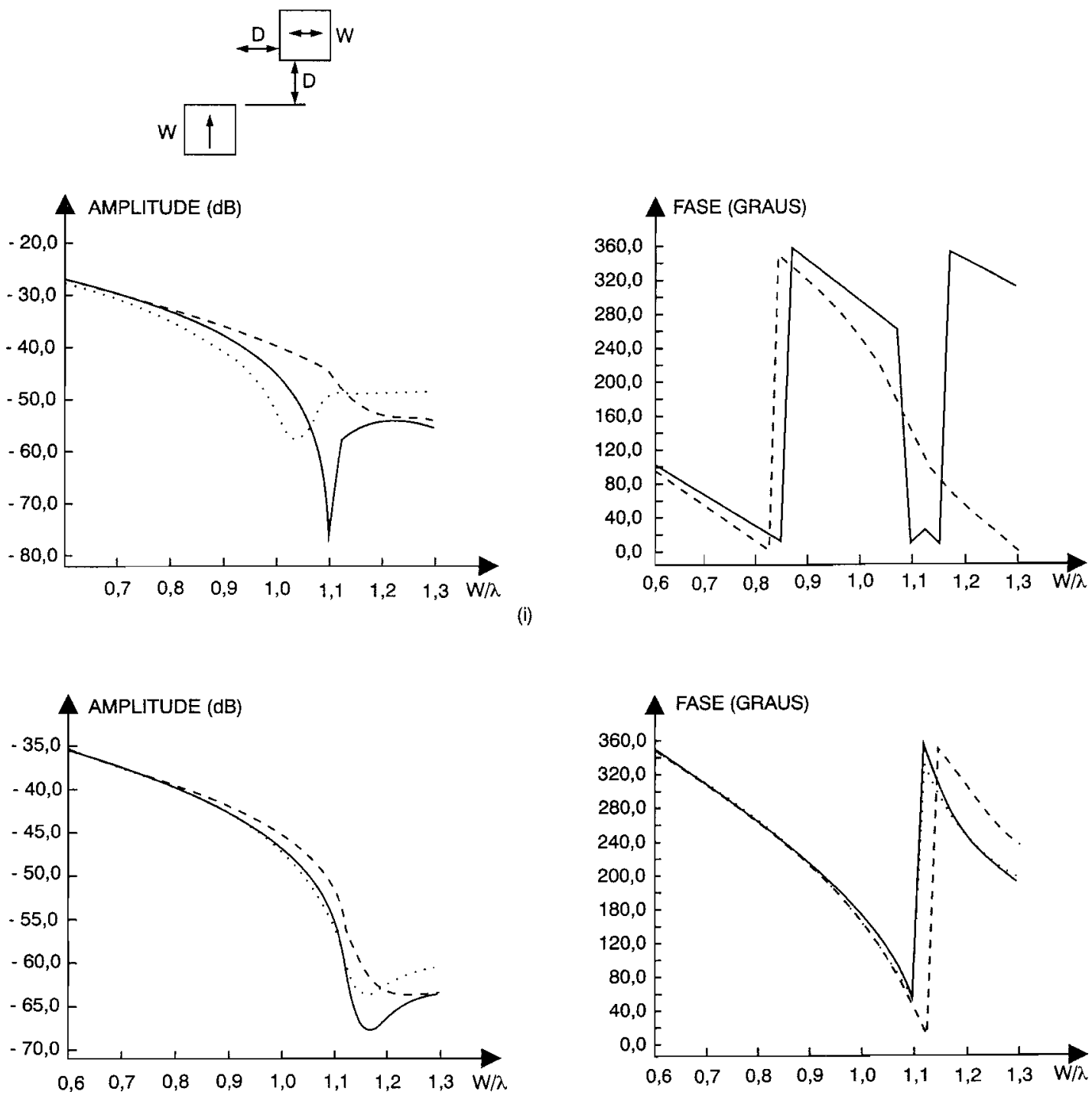

(ii)
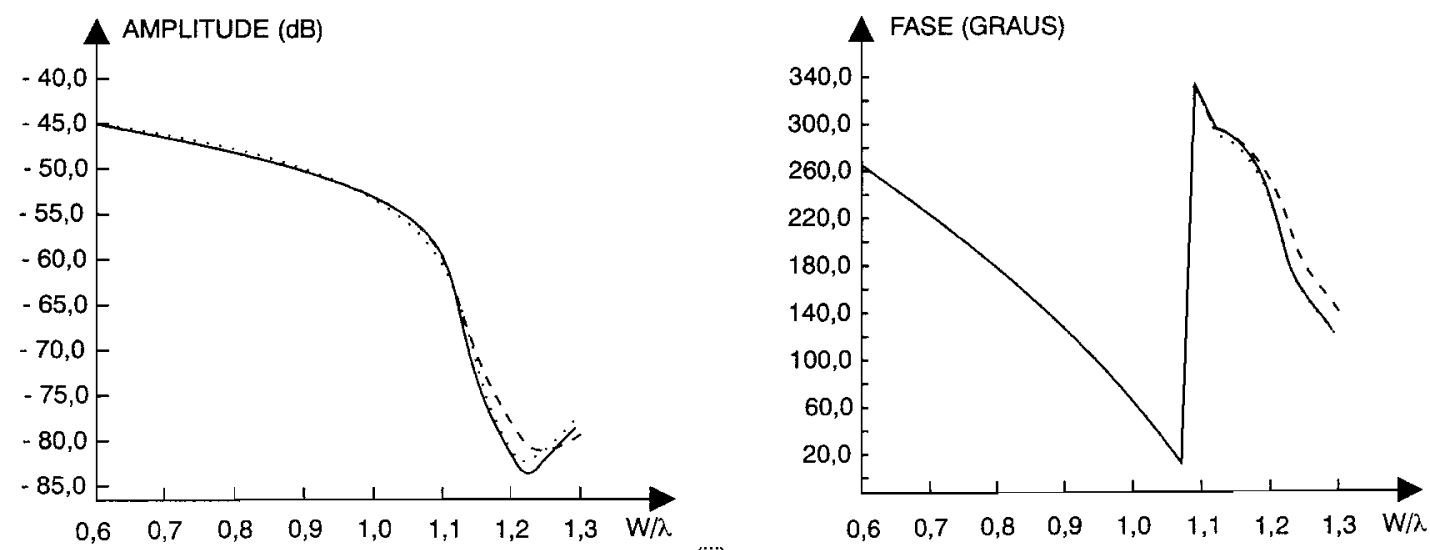

Fig. 3d - Acoplamento entre os modos $T E_{10}-T E_{01}$ (plano diagonal):

(i) $D=0,05 \lambda$; (ii) $D=1 \lambda$; (iii) $D=4 \lambda$ 
A determinação da componente de polarização cruzada radiada pelo conjunto requer a predição adequada do acoplamento entre modos ortogonais que aparecem quando as aberturas estão alinhadas fora do plano - E e - H. Este acoplamento é mais intenso quando as aberturas estiverem sobre o plano diagonal, sendo esta situação ilustrada pelo acoplamento entre os modos $\mathrm{TE}_{10} \mathrm{TE}_{01}$.

Os gráficos apresentados nas Figuras 3.a-d correspondem à amplitude e à fase do elemento da matriz [S] relacionada ao acoplamento entre os modos em questão. Deles podemos observar que o acoplamento mais forte ocorre ao longo do plano-E para pequenos valores de D. De um modo geral, a amplitude decai monotonicamente, até que os modos TE12 e TM12 (que se acoplam fortemente ao modo TE10) passam a ser propagantes. Quando comparadas a formulação integral, as maiores discrepâncias apresentadas pelas fórmulas assintóticas aparecem para o acoplamento ao longo do plano-E onde este é mais intenso. Para as demais posições, as discrepâncias são menores, visto que as regiões de campo mais intenso sobre as aberturas encontram-se mais afastadas que no caso anterior. Para aberturas adjacentes $(D=0,05 \lambda)$ ambas as expansões apresentam considerável discrepância dos resultados dados pela formulação integral. Para aberturas distantes de $\mathrm{D}=1 \lambda$, até a entrada dos modos superiores, a expansão Exp-1 (- - ) para o acoplamento no plano-E apresenta desvios menores que $5 \mathrm{~dB}$ para a amplitude e menores que $35^{\circ}$ para a fase, sendo menores que $1,5 \mathrm{~dB}$ e $12^{\circ}$ para Exp-2, respectivamente. Para as demais posições, Exp-2 apresenta uma excelente aproximação, especialmente na descrição da fase do acoplamento. Para aberturas mais afastadas, $\mathrm{D} \geq 2 \lambda$, a inclusão do termo de terceira ordem $\left({ }^{1} / \mathrm{R}_{\mathrm{ik}}{ }^{3}\right)$ na expansão permite descrever adequadamente o acoplamento além do ponto de entrada dos modos superiores. Para as aberturas sobre o plano diagonal, a expansão Exp-2 (.......) permite determinar o acoplamento com erro menor que $0,5 \mathrm{~dB}$ (ate ao nível de -60dB) e desvio de fase menor que 1 para valores de $\mathrm{D}=1 \lambda$.

A comparação entre os resultados da formulação integral e da expansão assintótica mostra que esta última pode ser aplicada com sucesso, mesmo para distâncias no entorno de $D=1 \lambda$, visto que as maiores discrepâncias (em $\mathrm{dB}$ ) ocorrem para valores muito baixos de acoplamento. Na prática, conjuntos de alimentadores retangulares com dimensões maiores que $0,6 \lambda$ são de grande interesse em sistemas de antenas refletoras para a produção de feixes modelados. Nestes casos, a expansão assintótica pode ser aplicada para o acoplamento entre as aberturas que não forem adjacentes entre si, quando teremos a condição de que $\mathrm{d} \geq 0,7 \lambda$, considerando uma separação típica de $0,05 \lambda$ entre aberturas. 


\section{Apêndice A}

\section{Formulação para Aberturas Retangulares}

\section{A.1 Método de Lewin (Aberturas Distintas)}

Uma vez conhecidos os modos que representarão os campos nas diversas aberturas retangulares, podemos resolver analiticamente duas das quatro integrais da equação (11) através do método de Lewin [2]. O resultado final é o seguinte:

$$
\begin{aligned}
& C_{m n}^{i j}=\frac{-j \pi \omega \varepsilon_{o}}{2\left(\lambda_{m} \lambda_{n}\right)^{2}} \frac{Z_{n}}{N_{m}} R_{m n}\left[C_{x} I_{x}+C_{y} I_{y}+C_{z} I_{z}\right] \\
& \underset{z}{\mathrm{I}} \underset{\mathrm{z}}{\mathrm{y}}=\frac{1}{16}\left\{\int _ { 0 } ^ { \mathrm { a } _ { \mathrm { j } } } \mathrm { d } \sigma \left[\int _ { 0 } ^ { \mathrm { b } _ { \mathrm { j } } } \mathrm { d } \lambda \left(\mathrm{~T}\left(-\sigma, \sigma_{1}, \sigma_{2} \mid-\lambda, \lambda_{1}, \lambda_{2}\right)+\mathrm{T}\left(\sigma_{3}, \sigma_{4},-\sigma_{4} \mid-\lambda, \lambda_{1}, \lambda_{2}\right)+\right.\right.\right. \\
& +\mathrm{T}\left(-\sigma, \sigma_{1}, \sigma_{2} \mid \lambda_{3}, \lambda_{4},-\lambda_{4}\right)+\mathrm{T}\left(\sigma_{3}, \sigma_{4},-\sigma_{4} \mid \lambda_{3}, \lambda_{4},-\lambda_{4}\right)+ \\
& \left.+\int_{0}^{b_{1}-b} \mathrm{~d} \lambda\left(\mathrm{T}\left(-\sigma, \sigma_{1}, \sigma_{2} \mid \lambda, \lambda_{1}, 2 \lambda+\lambda_{2}\right)+\mathrm{T}\left(\sigma_{3}, \sigma_{4},-\sigma_{4} \mid \lambda, \lambda_{1}, 2 \lambda+\lambda_{2}\right)\right)\right]+ \\
& +\int_{0}^{a_{1}-B} d \sigma\left[\int_{0}^{b_{j}} d \lambda\left(T\left(\sigma, \sigma_{1}, 2 \sigma+\sigma_{2} \mid-\lambda, \lambda_{1}, \lambda_{2}\right)+T\left(\sigma, \sigma_{1}, 2 \sigma+\sigma_{2} \mid \lambda_{3}, \lambda_{4},-\lambda_{4}\right)\right)+\right.
\end{aligned}
$$

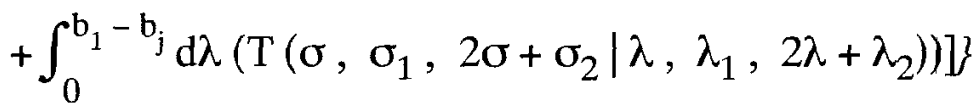

$$
\begin{aligned}
& T(A, B, C \mid D, E, F)=\underset{z}{\mathrm{~T}} \underset{z}{y}=G(\beta) \underset{+}{M+}+\left(A, B, C, d_{p}, s_{p}, a_{i}\right) \underset{+}{M+}+\underset{+}{+}\left(D, E, F, d_{q}, s_{q}, b_{i}\right) \\
& \beta=\left[\left(A+X_{i j}\right)^{2}+\left(D+Y_{i j}\right)^{2}\right]^{1 / 2} \\
& \mathrm{M}_{-}^{+}(\mathrm{A}, \mathrm{B}, \mathrm{C}, \mathrm{D}, \mathrm{E}, \mathrm{F})=\frac{2 \mathrm{~F}}{\pi}\left\{\frac{1}{\mathrm{D}} \operatorname{sen}\left[\frac{\mathrm{D} \pi(\mathrm{C}-\mathrm{B})}{2 \mathrm{~F}}\right] \cos \left[\pi\left(\mathrm{D}+\frac{\mathrm{AE}}{\mathrm{F}}+\frac{\mathrm{D}(\mathrm{B}+\mathrm{C})}{2 \mathrm{~F}}\right)\right] \pm\right.
\end{aligned}
$$




$$
\begin{gathered}
\quad \pm \frac{1}{E} \operatorname{sen}\left[\frac{E \pi(C-B)}{2 F}\right] \cos \left[\pi\left(E+\frac{A D}{F}+\frac{E(B+C)}{2 F}\right]\right\} \\
s_{p}=\left(p_{m}+p_{n} a_{i} / a_{j}\right) / 2 \quad \sigma=x-x^{\prime}, \quad \lambda=y-y^{\prime} \\
d_{p}=\left(p_{m}-p_{n} a_{i} / a_{j}\right) / 2 \quad \sigma_{1}=\sigma-a_{i} \quad \lambda_{1}=\lambda-b_{i}
\end{gathered}
$$

onde $\xi_{i}=\left\{\begin{array}{l}1, i=0 \\ 2, i>0\end{array}, \xi_{i}\right.$ é a permissividade na abertura “ $i$ " e $a_{i}, b_{i}$ são as dimensões desta abertura. Os parâmetros $\mathrm{p}_{\mathrm{m}}, \mathrm{q}_{\mathrm{m}}$ referem-se aos modos " $\mathrm{m}$ " da abertura " $\mathrm{i}$ " e $\mathrm{p}_{\mathrm{n}}, \mathrm{q}_{\mathrm{n}}$ aos modos " $n$ " da " $\mathrm{j}$ "; $x, y$ são as coordenadas do modo na abertura " $i$ " e $x$ ',y' na “ $\mathrm{j}$ ”. função de Green $G$ e dada pela equação (3). Os parâmetros $X_{\mathrm{ij}}, \mathrm{Y}_{\mathrm{ij}}$ ) são mostrados na figura 2 e $R_{m n}, C_{x}, C_{y}, C_{z}$ são apresentados na tabela A.l. As integrais da equação (A.2) devem ser resolvidas numericamente. $\mathrm{O}$ método utilizado pelos autores para a resolução numérica destas integrais foi o da Quadratura Gaussiana.

\section{Tabela A.1}

Parâmetros $\mathrm{R}_{\mathrm{mn}}, \mathrm{C}, \mathrm{C}_{\mathrm{y}}, \mathrm{C}_{\mathrm{z}}$.

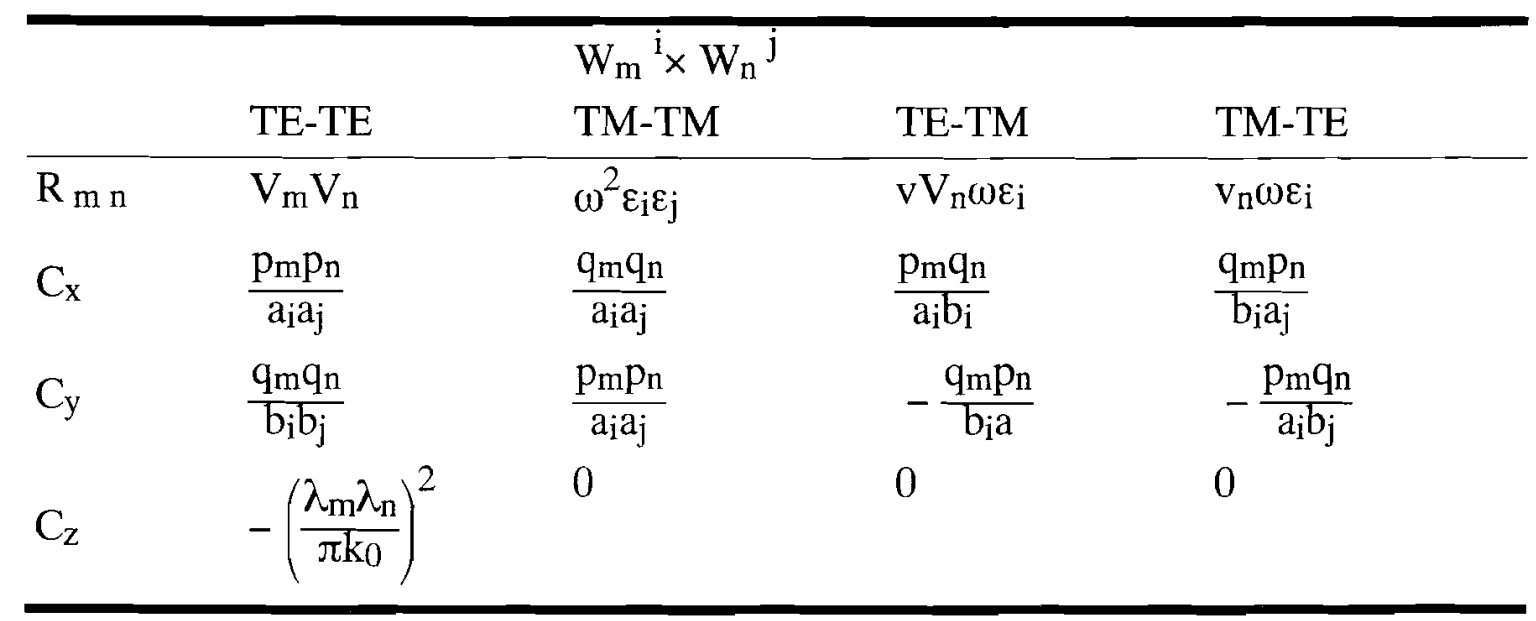




\section{A.2 Acoplamento entre Modos na Mesma Abertura}

Quando os modos " $\mathrm{m}$ ", " $\mathrm{n}$ " encontram-se na mesma abertura $\left(x_{i j}=y_{i j}=0, a_{i}=a_{j}, b_{i}=b_{j}\right)$, algumas simplificações podem ser feitas na equação (A.2), onde apenas restarão as integrais cujos limites vão de $O$ até $a_{j}$ ou $b_{j}$. Aplicando a seguinte transformação sobre as variáveis de integração o e $\lambda$ :

$$
\left\{\begin{array}{l}
\sigma=\rho \cos \psi \\
\lambda=\rho \operatorname{sen} \psi
\end{array} \Rightarrow \mathrm{d} \lambda \mathrm{d} \sigma=\rho \mathrm{d} \rho \mathrm{d} \psi\right.
$$

podemos não só resolver uma das integrais analiticamente como há o cancelamento da singularidade que existe, neste caso, em $\sigma=\lambda=0$. Os resultados finais são os seguintes:

$$
\begin{aligned}
\underset{\mathrm{Z}}{\mathrm{I}}= & \frac{\mathrm{a}_{\mathrm{i}} \mathrm{b}_{\mathrm{i}}}{4}\left[1+\cos \left(\mathrm{p}_{\mathrm{m}} \mathrm{pi}\right) \cos \left(\mathrm{p}_{\mathrm{n}} \mathrm{pi}\right)\right]\left[1+\cos \left(\mathrm{q}_{\mathrm{m}} \pi\right) \cos \left(\mathrm{q}_{\mathrm{n}} \pi\right)\right] \times \\
& \left\{\times \int_{0}^{\theta} G G \underset{z}{y}\left(s_{p}, d_{p}, s_{q}, d_{q}, \frac{\cos \psi}{a_{i}}, \frac{\operatorname{sen} \psi}{b_{i}}, \frac{a_{i}}{\cos \psi}\right) d \psi+\right. \\
& \left.+\int_{\theta}^{\frac{\pi}{2}} \mathrm{GG}_{\mathrm{z}}^{\mathrm{x}}\left(\mathrm{s}_{\mathrm{p}}, \mathrm{d}_{\mathrm{p}}, \mathrm{s}_{\mathrm{q}}, \mathrm{d}_{\mathrm{q}}, \frac{\cos \psi}{\mathrm{a}_{\mathrm{i}}}, \frac{\operatorname{sen} \psi}{\mathrm{b}_{\mathrm{i}}}, \frac{\mathrm{b}_{\mathrm{i}}}{\operatorname{sem} \psi}\right) \mathrm{d} \psi\right\}
\end{aligned}
$$

$\underset{\mathrm{z}}{\mathrm{GG}} \underset{\mathrm{y}}{\mathrm{x}}(\mathrm{b}, \mathrm{c}, \mathrm{d}, \mathrm{e}, \mathrm{f}, \mathrm{g})=\mathrm{FF}(\pi \mathrm{b}, \mathrm{e}, \pi \mathrm{d}, \mathrm{f}, \pi \mathrm{a}, \mathrm{pic}, \mathrm{g}) \underset{+}{+} \mathrm{FF}(\pi \mathrm{b}, \mathrm{e}, \pi \mathrm{c}, \mathrm{f}, \pi \mathrm{a}, \pi \mathrm{d}, \mathrm{g})+$

$$
\overline{+} F F(\pi a, e, \pi d, f, \pi b, \pi c, g)-F F(\pi a, e, \pi c, f, \pi b, \pi d, g)
$$

onde $\theta=\tan ^{-1}\left(b_{i} / a_{i}\right)$. A função FF e dada por: 


\section{Para $A \neq 0$ e $C \neq 0$ (argumentos de FF):}

$$
\begin{aligned}
& F F(A, B, C, D, E, F, G)=\frac{1}{8 A C}[I 1(2(A-C), B(E-A)-D(F-C), G)- \\
& -I 1(2(A+C), B(E-A)+D(F-C), G)-I 1(2 A, B(E-A)-D(F+C), G)+ \\
& +I 1(2 A, B(E-A)+D(F+C), I 1(2 C, D(F-C)-B(E+A), G)+\quad(A .10) \\
& I 1(2 C, D(F-C)+B(E+A), G)+I 1(0, B(E+A)-D(F+C), G)- \\
& -I 1(0, B(E+A)+D(F+C), G)] \\
& \text { I } 1(a, b, c)=\int_{0}^{C} \cos (a+b \rho) e^{-j ~ k_{0} \rho} d \rho
\end{aligned}
$$

\section{Para $A=0$ e $C \neq 0$ (argumentos de FF):}

$$
\begin{aligned}
& \text { FF }(A, B, C, D, E, F, G)=\frac{1}{4 C}[I 2(2 C, D(F-C)+B E, B, G)+I 2(2 C, D(F-C)-B E, B, G)- \\
& -I 2(0, D,(F+C)+B E, B, G)-I 2(0, D,(F+C)-B E, B, G)] \\
& I 2(a, b, c, d)=\int_{0}^{d}(1-c \rho) \operatorname{sen}(a+b \rho) e^{-j k_{0} \rho} d \rho
\end{aligned}
$$

\section{Para $A \neq 0$ e $C^{\prime}=0$ (argumentos de FF):}

É igual ao caso anterior, bastando trocar entre si os membros dos pares C,A; D,B; F,E.

Para $A=C=0$ (argumentos de FF):

$F F(A, B, C, D, E, F, G)=\frac{1}{2}[I 3(B E+D F, B, D, G)+I 3(B E-D F, B, D, G)]$

$$
I 3(a, b, c, d)=\int_{0}^{d}\left[1-(b+c) \rho+b c \rho^{2}\right] \cos (a \rho) e^{-j k_{0} \rho}
$$


As integrais das equações (A.11), (A.13) e (A.15) podem ser resolvidas analiticamente e as da equação (A.8) podem ser resolvidas numericamente através do método da Quadratura Gaussiana.

\section{Referências}

[1] T. S. Bird; "Mode Coupling in a Planar Circular Waveguide Array"; IEE J. Microw. Opt. Acoust. (MOA), v. 3, no. 5, pp. 172-180, 1979.

[2] T. S. Bird; "Analysis of Mutual Coupling in Finite Arrays of Different-Sized Rectangular Waveguides"; IEEE Trans. Antennas Propagat.,V.AP-38,no. 2, pp.166172,1990

[3] D. Kitchener, K.Raghavan,C.G.Parlii;'’ Mutual Coupling in a Finite Planar Array of Rectangular Apertures", Electronic Letters, V.AP-23, No.21, pp. 11691170,1987 .

[4] Amitay, H. and Gans, M.J.;"Design of Rectangular Horns Array with Oversized Aperture Elements"; IEEE Trans. Antennas and Propagation, V.AP29,no.6,pg.871-884, 1981.

[5] Silver, S.; "Microwave Antenna Theory and Design"; Capítulo 7, Radiation Laboratory Series 12, McGraw-Hill, 1949.

[6] Moreira, F. J. S.; "Conjunto de Alimentadores para Antenas Refletoras com Feixes Modelados"; Dissertação de Mestrado, Departamento de Engenharia Elétrica, PUC/Rio, 1992.

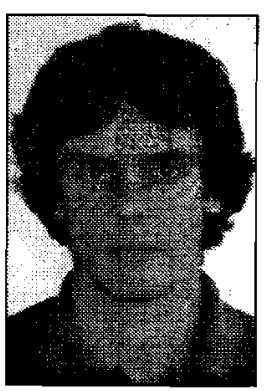

Fernando José da Silva Moreira nasceu no Rio de Janeiro em 18 de julho de 1967. Graduou-se em Engenharia Elétrica pela Pontifícia Universidade Católica do Rio de Janeiro em dezembro de 1989. Recebeu o título de mestre em Engenharia Elétrica (MSc.) pela Pontifícia Universidade Católica do Rio de Janeiro em julho de 1992. Está atualmente vinculado a University of Southern California onde desenvolve trabalho de pesquisa para sua tese de Doutorado. Seus principais interesses são eletromagnetismo aplicado e antenas. 


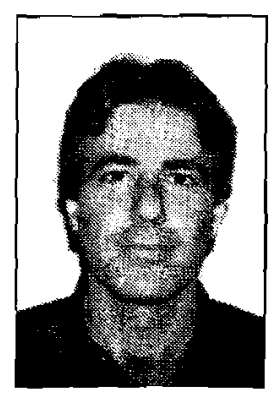

José Ricardo Bergmann nasceu em Porto Alegre em 25 de março de 1953. Graduou-se em Engenharia Elétrica pela Universidade Federal do Rio Grande do Sul em dezembro de 1975. Recebeu o título de Mestre em Engenharia Elétrica (MSc) pelo Instituto Militar de Engenharia em Fevereiro de 1979 e o título de Doutor em Engenharia Elétrica (PhD) pela University of London em Dezembro de 1986. É professor associado da Universidade Católica do Rio de Janeiro, estando vinculado a esta universidade desde 1979. Seus principais interesses em pesquisa são eletromagnetismo aplicado, síntese e análise de antenas refletoras e antenas para comunicaçóes via satélite. É atualmente presidente da Seção Rio de Janeiro do IEEE. 